\title{
Stacking Blocks and Counting Permutations
}

\author{
Lara K. Pudwell \\ Valparaiso University \\ Valparaiso, Indiana 46383 \\ Lara.Pudwell@valpo.edu
}

In this paper we will explore two seemingly unrelated counting questions, both of which are answered by the same formula. In the first section, we find the surface areas of certain solids formed from unit cubes. In second section, we enumerate permutations with a specified set of restrictions. Next, we give a bijection between the faces of the solids and the set of permutations. We conclude with suggestions for further reading. First, however, it is worth explaining how this paper came about.

The author received an email from David Harris while he was helping his 12-year-old daughter complete a project for her math class. Together Harris and his daughter constructed triangular piles of cubes. After creating an increasing sequence of these piles, they computed the surface area of each pile, and hoped to find a formula for the surface area of their $n$th pile. This project and its solution are described in the next section. At the time of their correspondence, Harris and his daughter had deduced several facts about the construction but were unable to find a formula for the surface area in general. When they searched for the first few terms in their sequence, Google returned only one hit: a Maple data file on the author's website.

The sequence that Harris and his daughter discovered online was originally generated in the context of pattern-avoiding words and permutations. Their web search produced a conjecture that gives a nice geometric interpretation of a permutation patterns question. This serendipitous discovery of the surprising and beautiful connection between a geometry problem and an enumeration problem illustrates how attractive new results may sometimes appear in such a surprising place as an elementary homework exercise. 

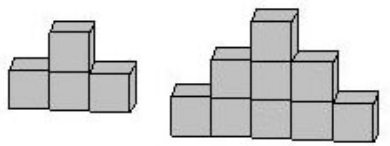

Figure 1: The second and third solids

\section{The Surface Area of Cubes}

We begin with the Harrises' original geometry question. We first describe a recursive construction involving unit cubes, and then compute the surface area of the $n$th solid in this construction.

The first solid is a unit cube, which has surface area 6 . To construct the $n$th solid, first form a row of $2 n-1$ cubes. Then, center the $(n-1)$ st construction on top of this row. For example, the second solid is shown in Figure 1. It has surface area 18. The third solid is also shown. It has surface area 34 .

Now, we wish to compute the surface area $S A_{n}$ of the $n$th solid. We have already computed $S A_{1}, S A_{2}$, and $S A_{3}$ above.

Notice that to construct $S A_{n}$, we glue together a solid of surface area $S A_{n-1}$ together with a rectangular prism of surface area $4 \cdot(2 n-1)+2=$ $8 n-2$. However, there are $2 n-3$ squares which overlap, and are now on the interior of the shape. Thus, the surface area only increases by $(8 n-$ $2)-2(2 n-3)=4 n+4$ units; that is $S A_{n}-S A_{n-1}=4 n+4$. Since the difference sequence for $S A_{n}$ is linear, we know that $S A_{n}$ is quadratic. Three points determine a quadratic, so we already have enough information to compute $S A_{n}$ in general. Let $S A_{n}=a n^{2}+b n+c$. We easily see that $S A_{n}-S A_{n-1}=(2 a) n+(b-a)$. Thus $2 a=4$ and $b-a=4$, or $a=2$ and $b=6$. Together with the fact that $S A_{1}=6$, we see that $S A_{n}=2 n^{2}+6 n-2$. 


\section{Permutation Patterns}

We have proved that the surface area of the Harrises' $n$th solid is $2 n^{2}+6 n-2$. We now give the necessary definitions to produce a set of permutations with $2 n^{2}+6 n-2$ elements.

Given a string of numbers $s$, the reduction of $s$ is the string obtained in the following way: find the smallest number in the string and replace all occurrences of that number with 1 , then find the second smallest number in the string and replace all occurrences of that number with 2, and so forth, replacing the occurrences of the $i$ th smallest number with the number $i$. For example, the reduction of 2671165 is 2451143. Now, given strings of numbers $p=p_{1} \cdots p_{n}$ and $q=q_{1} \cdots q_{m}$, we say that $p$ contains $q$ as a pattern if there exist indices $1 \leq i_{1}<i_{2}<\cdots i_{m} \leq n$ such that $p_{i_{1}} \cdots p_{i_{m}}$ reduces to $q$. Otherwise, we say that $p$ avoids $q$. For example, 2671165 contains the pattern 2321 because it contains the subsequence 6765 , which reduces to 2321. However, 2671165 avoids the pattern 1234 because it has no strictly increasing subsequence of length 4.

Finally, we introduce a bit of notation. In this paper we are concerned with permutations that have two copies of each letter. Given a set of permutations $Q$, let $S_{n}^{(2)}(Q)$ denote the set of permutations of two 1's, two 2 's, and so on up to two $n$ 's avoiding all patterns in the set $Q$. For example $S_{2}^{(2)}(\{112\})=\{1221,2121,2211\}$. Typically, a permutation refers to an ordering of $n$ distinct letters. Since we are considering permutations where there are more than one copy of each letter we may refer to our permutations as multiset permutations.

We now have the machinery necessary to state and prove a useful lemma. This lemma is a special case of a result of Burstein [3].

Lemma $1\left|S_{n}^{(2)}(\{132,231,213\})\right|=2 n+2$ for $n \geq 2$.

Proof. Since we will only consider permutations that avoid the set of patterns $\{132,231,213\}$ in this proof, we will write $A_{n}$ instead of $S_{n}^{(2)}(\{132,231$, $213\})$. 
Because no string of 1's and 2's will contain a pattern in $\{123,231,213\}$, we have that $A_{2}=\{1122,1212,1221,2112,2121,2211\}$, and $\left|A_{2}\right|=6$, as desired.

We proceed by induction. Consider $p \in A_{n}$. Let $p^{\prime}$ be the multiset permutation formed by deleting the two copies of $n$ in $p$. For example if $p=$ 312123 , then $p^{\prime}=1212$. Notice that since $p \in A_{n}$, we have that $p^{\prime} \in A_{n-1}$.

Now, given $p^{\prime} \in A_{n-1}$, we consider all the ways to insert two copies of $n$ into $p^{\prime}$ to obtain a multiset permutation in $A_{n}$. Notice that if $n$ is inserted between two letters of $p^{\prime}$, we have necessarily created either a 132 pattern or a 231 pattern. Thus, the $n$ 's can be inserted in one of only 3 ways: (i) both $n$ 's are prepended to the beginning of $p^{\prime}$, (ii) both $n$ 's are appended to the end of $p^{\prime}$, or (iii) one $n$ is prepended to the beginning of $p^{\prime}$ and the other $n$ is appended to the end of $p^{\prime}$. Clearly, (i) will always produce a member of $S_{n}^{(2)}$, however, (ii) and (iii) must be considered more carefully. In particular, appending an $n$ to the end of $p^{\prime}$ will only produce a 213-avoiding multiset permutation if $p^{\prime}$ avoids the pattern 21, i.e. if $p^{\prime}$ is weakly increasing. Thus, $\left|A_{n}\right|=\left|A_{n-1}\right|+2$, since we may prepend two $n$ 's to the beginning of any member of $A_{n-1}$, but we may also append two $n$ 's to the end of the unique increasing permutation of $A_{n-1}$, or we may prepend an $n$ to the beginning of it and append an $n$ to the end of it.

Finally, since $\left|A_{n}\right|-\left|A_{n-1}\right|=2$, we know that $\left|A_{n}\right|$ grows linearly, and use the fact that $A_{2}=6$ to compute the formula $\left|A_{n}\right|=2 n+2$.

This lemma is key to our main theorem, which is given at the end of the next section.

\section{A Bijection}

We now give a bijection between the faces of their $n$th solid of the Harrises' construction and the multiset permutations of $S_{n+1}^{(2)}(\{132,231,2134\})$. While we could count the permutations of $S_{n+1}^{(2)}(\{132,231,2134\})$ directly, a bijection not only will show that the two quantities in question are equal, but a bijection will also illuminate some parallels between the cube construction 


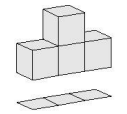

(i) (ii)
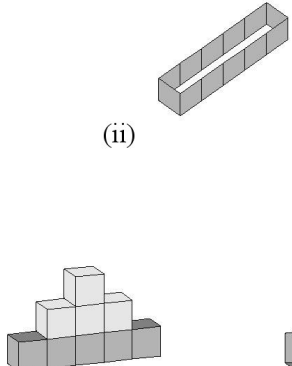

(iv)

(iii)
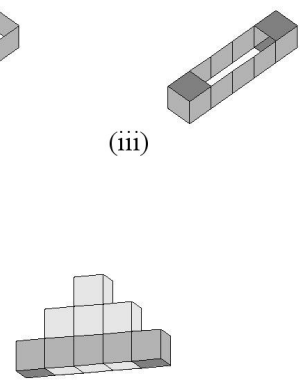

.

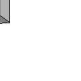

政

Figure 2: Constructing the $n=3$ solid from the $n=2$ solid

and the structure of the members of $S_{n+1}^{(2)}(\{132,231,2134\})$. To find such a bijection, it suffices to associate each permutation in $S_{n+1}^{(2)}(\{132,231,2134\})$ with a unique unit square on the surface of the Harrises' $n$th solid.

To this end, we consider another description of the Harrises' construction. To construct the $n$th solid from the $(n-1)$ st solid, we first remove the bottom face of the solid and move it one unit lower as in Figure 2 (i). Next, we form a rectangular ring of $4 n$ squares. This ring should be constructed so that it has two opposing sides of length 1 and two opposing sides of length $2 n-1$, as shown in Figure 2 (ii). Now, attach a new square to the top and bottom of each end of the ring, as shown in Figure 2 (iii), for a total of $4 n+4$ new squares. We may glue the modified version of the $(n-1)$ st solid together with this new modified ring of $4 n+4$ squares to form the $n$th solid. Two views of this gluing are shown in Figure 2 (iv).

This alternate construction has a clear advantage. Although it is more complicated to explain, this revised description allows us to associate each square on the surface of the $(n-1)$ st solid with squares on the $n$th solid, rather than "gluing" some squares into the interior.

The permutations of $S_{n+1}^{(2)}(\{132,231,2134\})$ also have a nice recursive 
structure. Given $p^{\prime} \in S_{n}^{(2)}(\{132,231,2134\})$, there are three ways to insert two copies of $(n+1)$ into $p^{\prime}$ to obtain a multiset permutation in $S_{n+1}^{(2)}(\{132,231$, 2134\}): (i) both $(n+1)$ 's are prepended to the beginning of $p^{\prime}$, (ii) both $(n+1)$ 's are appended to the end of $p^{\prime}$, or (iii) one $(n+1)$ is prepended to the beginning of $p^{\prime}$ and the other $(n+1)$ is appended to the end of $p^{\prime}$. As with the permutations of Lemma 1, (i) will always produce a member of $S_{n+1}^{(2)}(\{132,231,2134\})$, but (ii) and (iii) must be considered in more detail. In particular, appending $(n+1)$ to the end of $p^{\prime}$ may induce a copy of a forbidden 2134 pattern if $p^{\prime}$ contains a 213 pattern.

Now, we may recursively define a bijection between the squares of the $n$th solid and the permutations of $S_{n+1}^{(2)}(\{132,231,2134\})$.

To begin, since there are 6 elements of $S_{2}^{(2)}(\{132,231,2134\})$, and 6 faces in a unit cube, we may assign each one of these permutations to a unique face of the cube.

Now, consider the $n$th solid, constructed as described in this section. In the $(n-1)$ st solid, each of the light gray squares was associated with some permutation $p \in S_{n}^{(2)}(\{132,231,2134\})$. Let each such square now be associated with the permutation $(n+1)(n+1) p \in S_{n+1}^{(2)}(\{132,231,2134\})$.

We must now account for the four dark gray squares (the tops and bottoms of the left and right cubes in the bottom row of the solid) and the $4 n$ medium gray squares (the side faces of all cubes in the bottom row of the solid). Clearly, these must correspond to the permutations of $S_{n+1}^{(2)}(\{132,231,2134\})$ that either begin and end with $(n+1)$ or that end with two copies of $(n+1)$. Notice that each of these permutations was formed by taking one of the $2 n+2$ permutations in $S_{n}^{(2)}(\{132,231,213\})$ and inserting two $(n+1)$ 's in one of the two ways just described. Thus the $4 n+4$ permutations of the form $p(n+1)(n+1)$ or $(n+1) p(n+1)$ where $p \in S_{n}^{(2)}(\{132,231,213\})$ are precisely the members of $S_{n+1}^{(2)}(\{132,231,2134\})$ that correspond to the $4 n+4$ dark gray and medium gray squares. We now have established a recursive bijection between the exterior faces of the Harrises' piles of cubes and the members of $S_{n+1}^{(2)}(\{132,231,2134\})$. This cor- 
respondence gives a combinatorial proof of the following theorem, which was first observed using the method of enumeration schemes found in [6].

Theorem $1\left|S_{n+1}^{(2)}(\{132,231,2134\})\right|=2 n^{2}+6 n-2$ for $n \geq 1$.

\section{For Further Reading}

In this paper we found a bijection between the squares on the faces of the Harrises' $n$th construction, and certain pattern-avoiding permutations. This bijection illustrates the nice and unexpected connection between a question of middle school geometry and enumerative combinatorics.

The interested reader may wish to learn more about other enumeration problems related to this paper. Permutations which avoid other permutations have been actively studied since the seminal paper of Simion and Schmidt [7]. They aid in the study of a number of combinatorial objects. A friendly introduction to permutation patterns can be found in [2]. The permutations in this paper, with precisely two copies of each letter, are a special case of multiset permutations in which there may be an arbitrary numbers of copies of each letter. More detailed work with pattern avoidance involving multiset permutations can be found in [1], [3], [5], and [6].

The bijection demonstrated in this paper illustrates one of several connections between the Harrises' cube constructions and pattern-avoiding permutations. To see another bijection that relies on different geometric and combinatorial properties, visit the author's website at http://faculty.valpo. edu/lpudwell/papers.html.

Acknowledgment Thank you to David Harris for inspiring this paper, and to Andrew Baxter and two anonymous referees for many valuable presentation suggestions. 


\section{References}

[1] M. Albert, R. Aldred, M.D. Atkinson, C. Handley, and D. Holton, Permutations of a multiset avoiding permutations of length 3, Europ. J. Combin. 22 (2001), 1021-1031.

[2] M. Bóna, Combinatorics of Permutations, Chapman \& Hall, 2004, Chapter 4, 129-173.

[3] A. Burstein, Enumeration of Words with Forbidden Patterns, Ph.D. Thesis, University of Pennsylvania, 1998.

[4] D. Harris, Unpublished Correspondence, June 4, 2009.

[5] S. Heubach, and T. Mansour, Avoiding patterns of length three in compositions and multiset permutations, Advances in Applied Mathematics 36(2) (2006), 156-174.

[6] L. Pudwell, Enumeration Schemes for Pattern-Avoiding Words and Permutations, Ph.D. Thesis, Rutgers University, 2008.

[7] R. Simion and F. W. Schmidt, Restricted permutations, European J. Combin. 6 (1985), 383-406. 